\title{
ESTUDO DE PARÂMETROS DA VENTILAÇÃO NATURAL PARA MAXIMIZAÇÃO DO CONFORTO TÉRMICO EM PAVILHÕES INDUSTRIAIS: SIMULAÇÕES NUMÉRICAS
}

\section{Study of parameters of natural ventilation to thermal comfort maximization in industrial pavilions: numerical simulations}

\author{
Enaira Hoffmann de Oliveira ${ }^{1}$, Moacir Kripka ${ }^{2}$, Acir Mércio Loredo-Souza ${ }^{3}$
}

Recebido em 16 de fevereiro de 2014; recebido para revisão em 22 de março de 2014; aceito em 08 de abril de 2014; disponivel on-line em 04 de junho de 2014.

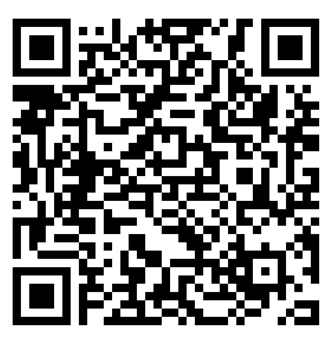

\section{PALAVRAS CHAVE:}

Pavilhões industriais; Ventilação natural; Conforto térmico.

\section{KEYWORDS:}

Industrial buildings, Natural ventilation, Thermal comfort

\section{* Contato com o autor:}

1 e-mail : arq.enaira@gmail.com Me, Programa de Pós-Graduação em Engenharia Civil e Ambiental - Universidade de Passo Fundo UPF

2e-mail :_mkripka@upf.br

${ }^{3}$ e-mail : 00009661@ufrgs.br
RESUMO: A ventilação natural é uma das estratégias de condicionamento natural que proporciona conforto térmico a um ambiente industrial. A otimização do sistema de ventilação natural em fábricas pode contribuir para a diminuição do consumo de aparelhos condicionadores de ar, trazendo benefícios ao meio ambiente e ao usuário. Este trabalho propõe a aplicação de técnicas de otimização para a determinação de parâmetros de conforto ambiental nos ambientes industriais. Busca-se maximizar a conforto térmico em pavilhões, com ou sem efeito do vento, tendo como variáveis de projeto as dimensões e as disposições das aberturas. A partir da simulação de diversas configurações de aberturas e dimensões de pavilhões, foram determinadas algumas relações como áreas das aberturas/área de piso e área da abertura de entrada/área da abertura de saída. Essas relações otimizadas foram comparadas com parâmetros indicados na literatura técnica.

\begin{abstract}
One of the strategies of natural conditioning that provides thermal comfort in an industrial environment is the natural ventilation. The optimization of natural ventilation systems in factories can contribute to reduce the consumption of air conditioners devices, bringing benefits to the environment and to the users. This work proposes the application of optimization techniques for the determination of parameters of environmental comfort in industrial buildings. In order to maximize the thermal comfort in pavilions, with or without considering the wind effect, the dimensions and arrangements of openings are taken as design variables. From the simulation of various configurations and dimensions of apertures flags, some relationships were determined such as areas of apertures versus floor area and inlet opening versus outlet opening area. These optimized relationships were compared to parameters indicated in the technical literature.
\end{abstract}

\section{( E. H. de Oliveira )}

( M. Kripka )

( A. M. Loredo-Souza )
Graduação em Engenharia Civil - Universidade Federal do Rio Grande do Sul - UFRGS 


\section{INTRODUÇÃO}

A preocupação com o aumento do consumo de energia para o condicionamento térmico das edificações e o bem estar de seus ocupantes incentiva a avaliação do desempenho ambiental dos edifícios. A ventilação natural propicia o condicionamento térmico dos ambientes e contribui para um bom desempenho ambiental dos edifícios. A movimentação natural do ar de forma adequada possibilita a renovação do ar no ambiente, além de diminuir a temperatura interna. Este tipo de estratégia de condicionamento térmico se faz através de aberturas (janelas, portas, lanternins, entre outros), que atuam como aberturas de entrada ou saída, e devem ser dimensionadas e estar posicionadas de modo a proporcionar um fluxo de ar adequado ao recinto.

A ventilação pode ser natural (espontânea) ou artificial (forçada). A ventilação natural se dá pela ação dos ventos (ventilação unilateral e ventilação cruzada), sendo o efeito chaminé ocasionado pela diferença de temperatura entre o ar interno e o ar externo. A ventilação artificial consiste na movimentação do ar por meios mecânicos (COSTA, 2005). Nos sistemas híbridos, os quais utilizam as duas formas de movimentação do ar, a ventilação é associada a equipamentos mecânicos, como ventiladores e condicionadores de ar, sendo possível reduzir o consumo de energia quando as condicionantes climáticas são favoráveis, sem afetar o conforto dos ocupantes.

A utilização da otimização no desempenho térmico de um ambiente é um dos objetivos mais relevantes do projeto arquitetônico (ROMERO, 2001). A otimização dos recursos energéticos por meio da redução dos custos com energia elétrica e seu consumo promovem alterações na forma como os edifícios são projetados, construídos e gerenciados.

O presente artigo tem como objetivo estudar a aplicação de técnica de otimização na maximização da ventilação natural em pavilhões industriais, fornecendo subsídios para a determinação da disposição e do dimensionamento das aberturas visando um maior conforto térmico com redução no consumo de energia.

\section{CONCEITOS BÁSICOS}

\subsection{VENTILAÇÃO NATURAL}

A ventilação natural se faz quando há deslocamento do ar dentro de um recinto fechado. A verificação do desempenho da ventilação natural esta associada à forma do edifício, ao microclima onde está inserido, a dimensão, o tipo, o número e a posição das aberturas nas edificações, seu entorno construído, bem como a velocidade do vento e diferenças de temperatura. dimensionamento da ventilação deve considerar o volume do ambiente, a quantidade de pessoas e a existência de equipamentos que geram calor, como computadores, máquinas e lâmpadas. Essas características, consequentemente, influenciam na localização, tamanho e modo de abrir das aberturas.

A ventilação natural pode ocorrer de duas formas: por meio do efeito dos ventos e pelo efeito chaminé. Entretanto, também podem ocorrer os dois mecanismos simultaneamente, dependendo das condições atmosféricas, do projeto e da localização do edifício. A ação dos ventos causa diferença de pressão sobre os edifícios e provoca a formação de zonas expostas a pressões positivas e de zonas expostas a pressões negativas. Há condição de ventilação do ambiente quando as aberturas de vãos em paredes sujeitas a pressões positivas possuem entrada de ar e as paredes sujeitas a pressões negativas possuem saída de ar.

Segundo Toledo (1999), a ventilação por efeito chaminé se dá quando há uma diferença de pressões originadas das diferenças de temperaturas do ar interno e externo do edifício. Assim, o ar interno, mais aquecido que o externo, sairá pelas aberturas altas, enquanto o ar externo, com temperatura inferior, entrará pelas aberturas baixas. Observa-se que o fluxo de ar será tanto mais intenso quanto mais baixas forem as aberturas de entrada e quanto mais altas forem as aberturas de saída do ar (FROTA, 2000).

\subsection{CÁlCULOS DE VAZÃo DE AR- MÉTOdOS EMPÍRICOS}

Os modelos empíricos de predição da 
ventilação são úteis por permitir uma rápida estimativa inicial das taxas de renovação do ar e da velocidade interna, todavia a aplicação deve atentar para as limitações (ALLARD, 2002). Há centenas de modelos empíricos desenvolvidos para estimar o desempenho da ventilação (CHEN, 2008). Os modelos mais simples fazem uso de equações que consideram o coeficiente de descarga da abertura, a velocidade do vento na altura de interesse, a área efetiva da abertura e os coeficientes de pressão sobre as fachadas.

Dentre os modelos empíricos estudados, apresenta-se o modelo de Clezar e Nogueira (1999), utilizado neste trabalho. O método é baseado na norma NBR 6123 (ABNT, 1987), que permite calcular a vazão de ar considerando o efeito chaminé, o efeito dos ventos, ou então, ambos os efeitos simultaneamente, através de uma formulação única. Além disso, esta formulação aplica-se a qualquer número e distribuição de aberturas.

A formulação é apresentada para o pavilhão indicado na Figura 1 , onde $\rho_{\mathrm{e}}$ e $\rho_{\mathrm{i}}$ representam as massas específicas do ar, correspondentes às temperaturas médias externas e internas, respectivamente. $A$ pressão $P_{0}$ será a pressão efetiva do ar interno na cota estabelecida como sendo o nível de referência. As pressões $\mathrm{P}_{\mathrm{e}, \mathrm{j}} \mathrm{e}$ $\mathrm{P}_{\mathrm{i}, \mathrm{j}}$ são as pressões efetivas junto à abertura “ "j", na cota $z_{j}$, correspondentes aos lados externo e interno, respectivamente.

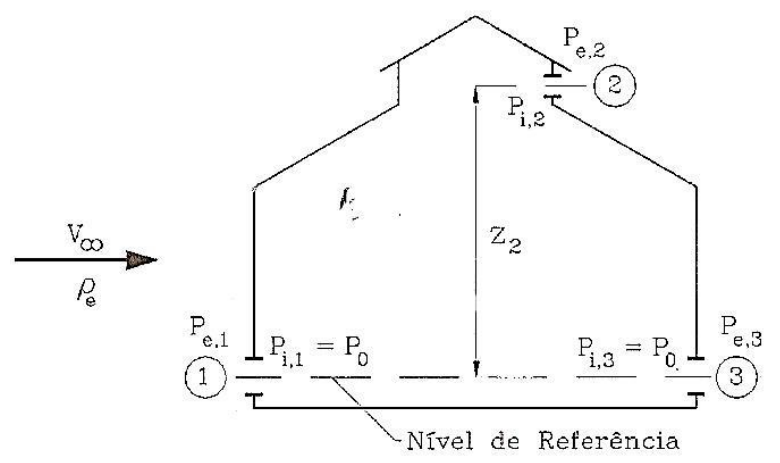

FIGURA 1: Representação das aberturas de uma edificação tipo galpão e parâmetros físicos do ar que influenciam no desempenho.

FONTE: Clezar e Nogueira (1999).
As hipóteses simplificativas, adotadas na formulação de Clezar e Nogueira (1999), são:

- Regime permanente: com esta hipótese, ignorase qualquer flutuação na intensidade e na direção do vento, desconsidera-se qualquer oscilação de temperatura externa e interna e não se levam em conta, também, possíveis alterações decorrentes do fechamento de algumas aberturas.

- Perda de carga no escoamento interno desprezível: esta hipótese é bastante razoável, tendo-se em vista a magnitude bastante grande da seção transversal implicando uma baixa velocidade do escoamento nestes ambientes.

\subsection{Otimização}

Otimização é a área da Matemática Aplicada que se preocupa em calcular e computar valores ótimos para variáveis de decisão que induzem desempenho ótimo, ao mesmo tempo em que satisfazem restrições, de um modelo matemático (CAMPONOGARA, 2006). Os métodos de otimização são utilizados quando se pretende minimizar ou maximizar uma função. Estes algoritmos procuram chegar a valores "ótimos" de forma diferenciada em problemas nos quais o cálculo analítico seria impraticável. De um modo geral, todos se utilizam de iterações, comparando os valores das anteriores com as novas, e através de algumas regras de decisão, escolhendo qual "direção" seguir.

A otimização permite que um determinado problema seja representado, de forma adequada, por meio de um modelo matemático, satisfazendo restrições especificas. Modelos matemáticos representam complexos sistemas físicos que dependem de soluções e recursos computacionais para a sua resolução. Há um grande interesse no desenvolvimento e implementação destes modelos em todas as áreas do conhecimento, tanto para que haja economia de materiais e de tempo como para o aumento da produtividade ou lucratividade dos recursos. Os modelos de programação matemática trabalham com processos de decisão e são compostos por 
uma função objetivo e por determinadas restrições, relacionadas a um conjunto de variáveis, descritas através de um conjunto de equações e inequações algébricas.

O estudo da aplicação da otimização nas análises do desempenho térmico foi abordado principalmente por autores internacionais, que de forma geral, descrevem os métodos numéricos e as variáveis de projeto determinando as condições de conforto térmico. Algumas das pesquisas mais recentes encontradas sobre a otimização aplicada ao conforto térmico são citadas a seguir.

O método descrito por Bouchlaghem (1990) simula o desempenho térmico do edifício, e aplica técnicas de otimização para determinar as melhores condições de conforto térmico. São consideradas as variáveis de projeto relacionadas à tipologia de edifícios, a orientação solar e a relação das aberturas, entre outros.

Caldas e Norford (2002) descrevem um método para encontrar as dimensões das janelas (largura e altura) que resultam num edifício com pequena quantidade de energia necessária para aquecimento e iluminação artificial. A otimização é baseada nos resultados de um software de simulação detalhada (Energy Plus). O software ajusta automaticamente a quantidade de iluminação artificial, de tal forma que a iluminação desejada seja alcançada.

O método descrito por Jedrzejuk e Marks (2002), decompõe o problema de projeto nos seguintes subproblemas: otimização de espaços internos, tipologia da construção e, finalmente, a coordenação das soluções. A forma do edifício está representada por meio de parâmetros como a altura das paredes, o número de pisos, a equivalência dos vãos da janela para com as áreas de parede, entre outros. O método é baseado em uma formulação que utiliza os custos da construção, a posição solar, a energia e a poluição emitida pelas fontes de calor, como funções objetivo.

Stephan (2009) desenvolveu um modelo baseado na dinâmica dos fluidos computacional com o objetivo de otimizar as taxas de ventilação natural, variando as dimensões das aberturas nas construções utilizando o software GenOpt. O autor calculou a altura da abertura a fim de otimizar tanto a taxa de fluxo de ar quanto o conforto térmico dos ocupantes. A função objetivo da formulação teve base nos critérios de conforto em relação ao clima do lugar, determinando assim as alturas das aberturas de acordo com a ventilação encontrada.

Trindade et al, (2010) investigaram formas de prever o comportamento da ventilação natural em edifícios por meio de ferramentas de simulação computacional, visando apoiar a tomada de decisão no projeto de arquitetura. Utilizaram um programa de dinâmica de fluidos computacional e um de simulação térmica e energética de edificações aplicada em galpões industriais. $\mathrm{O}$ artigo tem como foco a discussão dos procedimentos necessários para viabilizar as análises da ventilação, indicando como o método teve de ser adequado à complexidade de sucessivas abordagens, resultando em simplificações e incertezas de resultados.

De forma geral, o uso da simulação computacional como ferramenta de auxílio ao projeto se consolida acessível apenas a grupos de pesquisa de pequenos e médios portes. Esses programas ainda são praticamente desconhecidos da maioria dos projetistas atuantes no Brasil, uma vez que requerem para seu uso conhecimentos pouco difundidos na formação de arquitetos. Em países desenvolvidos, os grandes escritórios têm maiores possibilidades de uso dessas ferramentas, pois conseguem envolver equipes multidisciplinares para projetos de grande porte, ao contrário dos pequenos escritórios (PEDRINI e SZOKOLAY, 2005).

\section{FORMULAÇÃO DO PROBLEMA}

O principal parâmetro de um projeto de ventilação industrial é a vazão de ar, ou seja, a vazão volumétrica de ar necessária para o ambiente. $O$ dimensionamento das aberturas visa fornecer área suficiente para satisfazer as necessidades de ar dos usuários. Com a finalidade de avaliar a menor área de abertura necessária para manter o conforto térmico em uma 
edificação do tipo pavilhão industrial, foram consideradas como variáveis no problema, ilustradas na Figura 2, a altura do peitoril e da abertura tanto inferior quanto superior considerando os efeitos do vento e chaminé. Com a determinação da dimensão "altura" das aberturas, foi calculada a área da abertura e com a altura dos peitoris foi possível calcular a distância entre as aberturas. Como diretriz de projeto, para que haja coerência nas dimensões das fachadas, o peitoril mínimo também consistiu em um parâmetro fixo (Figura 3).

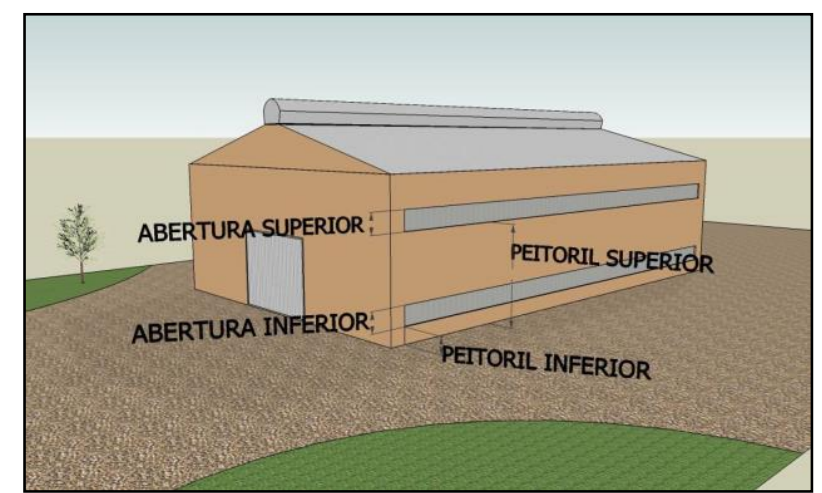

Figura 2: Pavilhão industrial com indicação das variáveis de projeto.

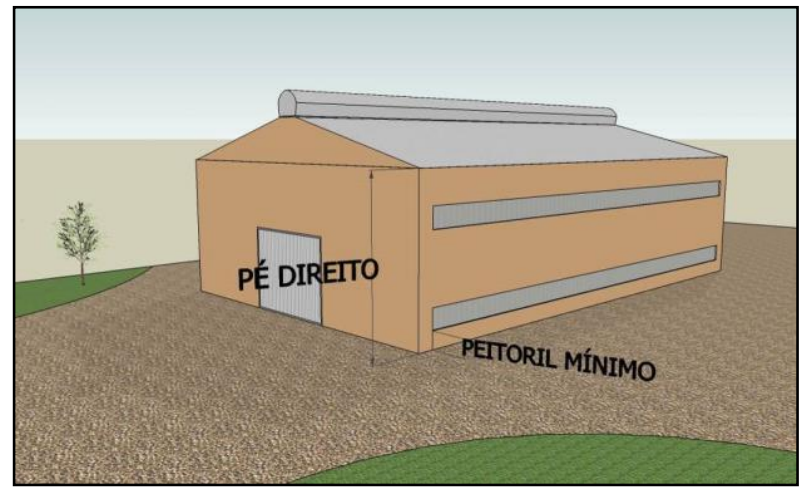

Figura 3: Pavilhão industrial com indicação da restrição da altura mínima do peitoril.

As restrições das alturas foram delimitadas de forma que os somatórios das dimensões de peitoril e altura das aberturas superiores fossem menores que a altura do pédireito do pavilhão (Figura 4).

$$
P_{S}+A_{S}<P_{d}
$$

Onde:

$\mathrm{P}_{\mathrm{s}}=$ peitoril superior (em metros);

$A_{s}=$ altura da abertura superior (em metros);

$P_{d}=$ pé-direito (em metros).

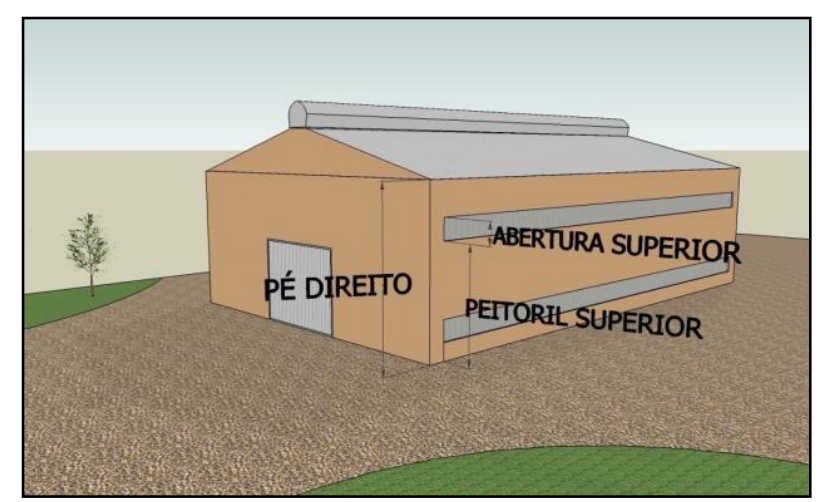

Figura 4: Pavilhão industrial com restrição relativa ao somatório das dimensões de peitoril e altura das aberturas superiores inferiores à altura do pé-direito do pavilhão.

Além disso, o somatório das dimensões do peitoril e da altura da abertura inferior não deve ultrapassar o peitoril da abertura superior (Figura 5).

$$
P_{i}+A_{i}<P_{s}
$$

Onde:

$\mathrm{P}_{\mathrm{i}}=$ peitoril inferior (em metros);

$A_{i}=$ altura da abertura inferior (em metros);

$\mathrm{P}_{\mathrm{s}}=$ peitoril superior (em metros).

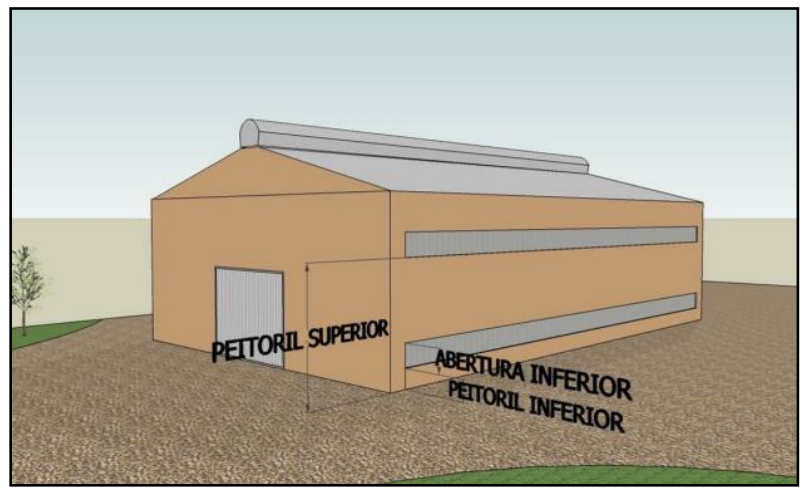

FIGURA 5: Pavilhão industrial com restrição relativa ao somatório das dimensões do peitoril e da abertura inferiores não ultrapassar o peitoril da abertura superior.

As dimensões das aberturas inferiores $\left(A_{i}\right)$ constituem as variáveis de projeto a serem determinadas. Desta forma, o problema abordado passa a ser formulado como:

$$
\min f\left(\mathrm{~A}_{\text {inf },}, \mathrm{A}_{\text {sup }}, \mathrm{P}_{\text {inf }}, \mathrm{P}_{\text {sup }}\right)=\left|\sum_{\mathrm{i}=1}^{\mathrm{n}_{\text {inf }}} \mathrm{A}_{\text {ent }_{\mathrm{i}}}+\sum_{\mathrm{j}=1}^{\mathrm{n}_{\text {sup }}} \mathrm{A}_{\text {sai }}\right| \text { Eq.[3] }
$$

Sujeito a:

$$
\begin{gathered}
\mathrm{Q}_{\text {nec }} \leq \mathrm{Q}_{\text {calc }} \\
\mathrm{P}_{\mathrm{s}(\mathrm{i})}+\mathrm{A}_{\mathrm{s(i)}}<\mathrm{P}_{\mathrm{d}} \quad \mathrm{i}=1, \mathrm{n}_{\text {sup }}
\end{gathered}
$$




$$
\begin{array}{cll}
P_{i(j)}+A_{i(j)}<P_{s} & j=1, n_{\text {inf }} & \text { Eq.[06] } \\
A_{\text {inf }}(i) \geq 0 & i=1, n_{\text {sup }} & \text { Eq.[07] } \\
A_{\text {sup }}(j) \geq 0 & j=1, n_{\text {inf }} & \text { Eq.[08] }
\end{array}
$$

Onde:

$n_{\text {sup }}$ é o número de aberturas superiores;

$n_{\text {inf }}$ o número de aberturas inferiores.

A formulação desenvolvida foi implementada no Microsoft Excel Solver, ferramenta escolhida por ser de ampla disponibilidade e fácil utilização. Para a determinação das vazões, foram empregadas as expressões da Norma Britânica BS 5925 (1980), da ASHRAE (2001), de Frota e Schiffer (2001), de Clezar e Nogueira (1999) e de Silvani (2005).

As planilhas desenvolvidas foram organizadas de forma esquemática em três partes:
(1) dados do problema (onde são inseridos tanto os dados de entrada como os valores iniciais para as variáveis), (2) descrição do problema (onde são descritos de forma sucinta os objetivos, as variáveis e as restrições), e (3) resolução do problema (onde são apresentados os valores da vazão necessária e a função objetivo para a solução atual).

Cabe destacar que, apesar de direcionada a pavilhões industriais, a metodologia proposta neste trabalho pode ser empregada para edificações com diferentes finalidades, como edifícios institucionais (escolas, unidades de saúde) e residências, sendo necessária a configuração das ferramentas utilizadas conforme a tipologia escolhida.

Na Figura 6 é representada uma das

\begin{tabular}{|c|c|}
\hline \multicolumn{2}{|l|}{ VARIÁVEIS } \\
\hline \multicolumn{2}{|l|}{ ALTURA DOS PEITORIS } \\
\hline \multicolumn{2}{|l|}{ ALTURA DAS ABERTURAS } \\
\hline \multicolumn{2}{|l|}{ RESTRIÇÕES } \\
\hline \multicolumn{2}{|l|}{ VAZÃONECESSÁR LA<=VAZÃO CALCULADA } \\
\hline ALTURA MÍNIMA DO PEITORIL INFERIOR & 1 \\
\hline$E\left(P_{s p}+A_{2 v p l}<P D\right.$ & 10,23002 \\
\hline$E\left(P_{\text {irf }}+A_{\text {irf }}\right)<P_{2 p}$ & 2,7300198 \\
\hline PD & 8 \\
\hline
\end{tabular}
planilhas desenvolvidas, empregando as expressões da ASHRAE (2001).

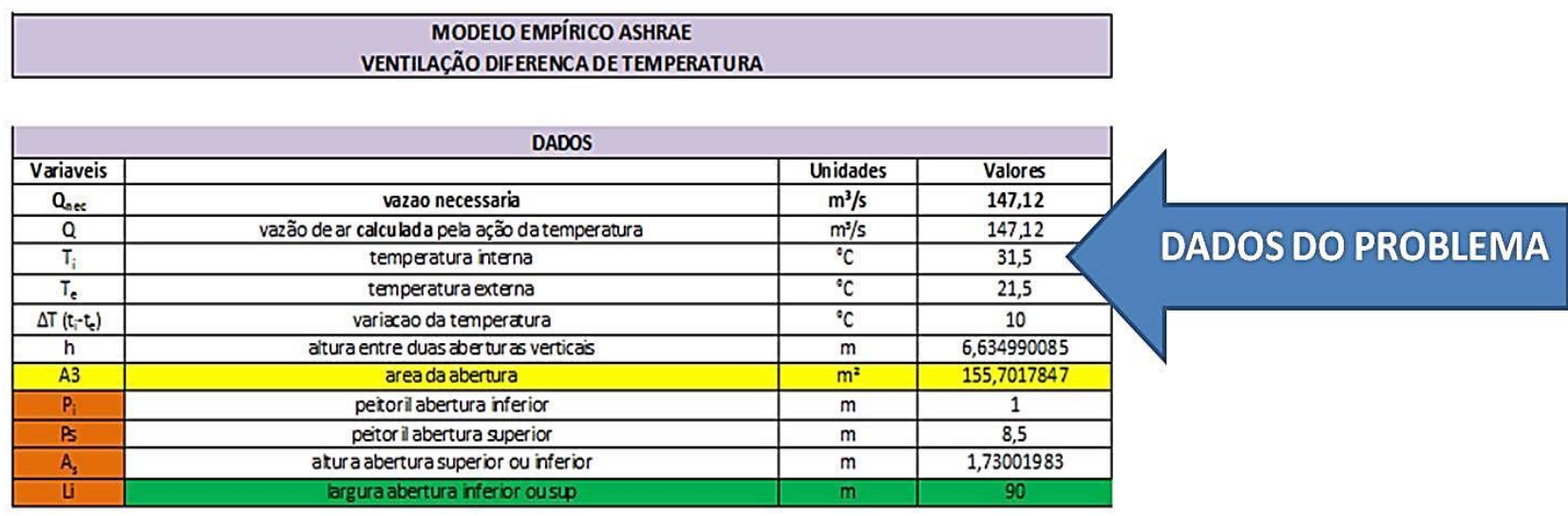
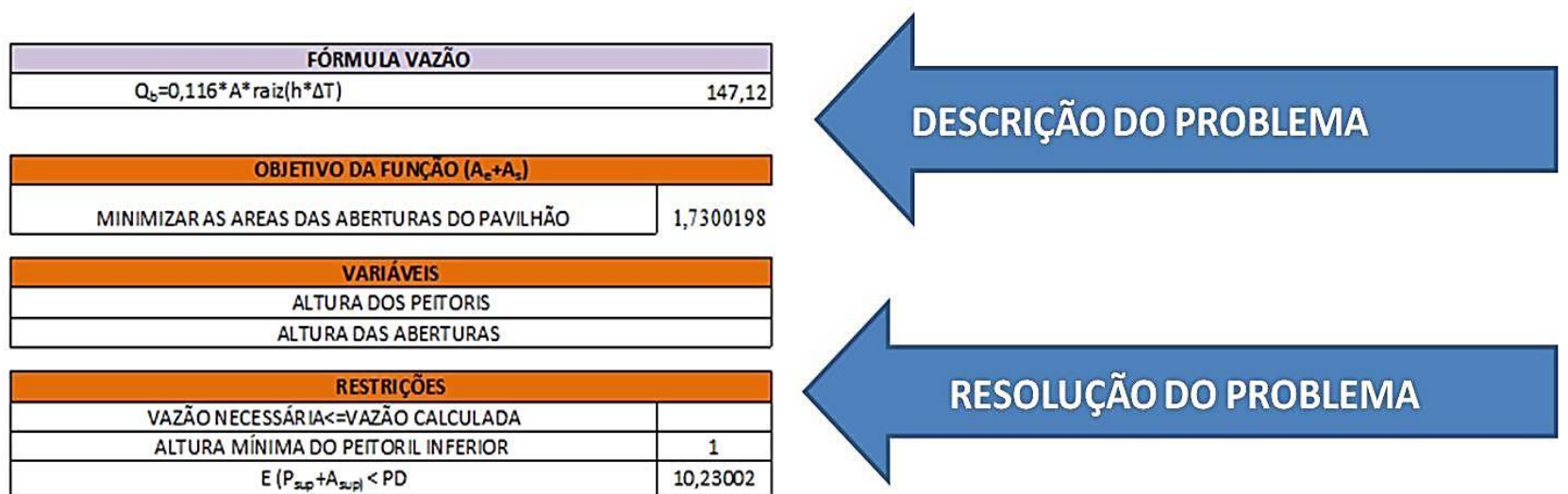

FIGURA 6: Exemplo de Planilha de Cálculo. 


\section{APLICAÇÕES DO MÉTODO}

A metodologia adotada para o desenvolvimento da pesquisa iniciou-se com base na simulação de modelos de pavilhões representativos com diferentes combinações de dimensões, largura, comprimento e tamanhos de aberturas, considerando o mesmo entorno. 0 objetivo consistia em avaliar a influência de cada variável no desempenho da ventilação. Conhecendo-se a atuação das variáveis tornou-se possível estabelecer uma lista de prioridades a serem atendidas no início da fase projetual potencializando a ventilação natural da edificação.

$\mathrm{Na}$ primeira etapa foi realizado o estudo de um determinado pavilhão industrial considerando as características dos componentes das aberturas, a velocidade e direção do vento, a diferença de temperatura e vazão. Esta etapa validou a formulação descrita pelo método estudado, e identificou a importância do cálculo da vazão nas dimensões ótimas obtidas. Este estudo utilizou dados da NBR 6123 (ABNT, 1988) e valores obtidos por Silvani (2005). Em seguida, utilizando a simulação computacional, foi verificado como as dimensões e as disposições das aberturas nas fachadas influenciam a vazão do local. Nestas simulações, os pavilhões foram modelados com e sem ventilação natural. Este método engloba de forma clara os dados analisados e permite a identificação das aberturas de entrada e saída através dos coeficientes. Assim, as edificações especificadas no trabalho podem ter aberturas de áreas iguais ou não e com diferentes posições, mas com iguais coeficientes de descarga. A vazão do ar externo necessária, $Q_{e}$, foi calculada com base no número de trocas de ar no ambiente e no volume do pavilhão em questão.

\subsection{APLICAÇÕES DO MÉTODO- PRIMEIRA ETAPA}

O modelo de pavilhão utilizado nesta etapa, onde foram realizadas as aplicações das formulações bem como nos ensaios no túnel de vento, possui $50 \mathrm{~m}$ de largura e $100 \mathrm{~m}$ de comprimento, $8 \mathrm{~m}$ de pé-direito livre (desnível entre o piso e a face inferior da viga de cobertura) e um exaustor natural de cumeeira de $86 \mathrm{~m}$ de extensão. Possui três aberturas, duas de ventilação de entrada (abertura inferior) e uma de ventilação de saída (abertura superior, chaminé), não contabilizando os portões (Figura 7).

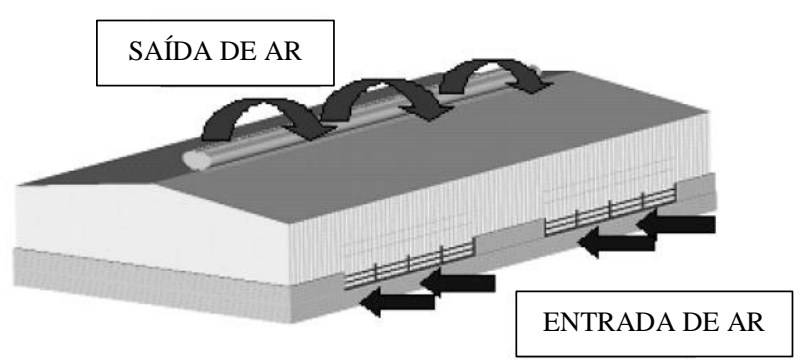

FIGURA 7: Modelo de pavilhão industrial empregado nas análises.

Para as análises foram atribuídos valores fixos de projeto retirados de Silvani (2005) para carga térmica, temperatura interna e externa, velocidade do vento, coeficientes de descarga e dimensões do exaustor de cumeeira.

- $\Delta \mathrm{t}=10^{\circ} \mathrm{C}$ (diferença de temperatura do ar interno e externo);

- $\mathrm{t}_{\mathrm{e}}=21,5^{\circ} \mathrm{C}$ (temperatura do ar externo);

- $Q_{n e c}=147,12 \mathrm{~m}^{3} / \mathrm{s}$ (vazão de ar necessária);

- $\rho_{\mathrm{e}}=1,20 \mathrm{~kg} / \mathrm{m}^{3}$ (massa específica do ar externo);

- $\mathrm{C}_{\mathrm{p}}=0,24 \mathrm{kcal} / \mathrm{kg}$ oC (calor específico do ar a pressão constante);

- $\rho \mathrm{i}=1,16 \mathrm{~kg} / \mathrm{m}^{3}$ ( massa específica do ar interno);

Os coeficientes de pressão para paredes e cobertura das diferentes zonas do galpão industrial, considerados nos cálculos foram obtidos através da NBR 6123 (ABNT, 1986) - Forças devidas ao vento em edificações para vento à $900,+0,7$ e $-0,5$, agindo perpendicularmente às fachadas principais. Para o efeito do vento, foram utilizados coeficientes de pressão interna e externa a $90^{\circ}$.

$\mathrm{Na}$ Tabela 1 são apresentados os resultados obtidos através aplicação direta e da otimização segundo a formulação proposta. Podese observar que os valores otimizados estão próximos aos determinados pela aplicação dos métodos. Foram considerados nos cálculos áreas das aberturas iguais, com única variável, $A_{i}=A_{s}$, relativa à altura da abertura superior e/ou inferior.

Da mesma forma, foram encontrados os resultados considerando 0 efeito chaminé (Tabela 2). 
TABELA 1: Áreas de entrada e saída de ar considerando o efeito do vento.

\begin{tabular}{|c|c|}
\hline \multicolumn{2}{|c|}{ Método de Clezar e Nogueira } \\
\hline Resultados das áreas necessárias & $48,25 \mathrm{~m}^{2}$ \\
\hline Resultados otimizados das áreas necessárias & $44,92 \mathrm{~m}^{2}$ \\
\hline
\end{tabular}

\begin{tabular}{|c|c|}
\hline \multicolumn{2}{|c|}{ TABELA 2: Áreas de entrada e saída de ar considerando o efeito chaminé. } \\
\hline \multicolumn{2}{|c|}{ Método de Clezar e Nogueira } \\
\hline Resultados das áreas necessárias & $150,50 \mathrm{~m}^{2}$ \\
\hline Resultados otimizados das áreas necessárias & $129,00 \mathrm{~m}^{2}$ \\
\hline
\end{tabular}

A relação entre as áreas necessárias e as áreas encontradas com o uso do solver variaram em torno de $16 \%$. A Figura 8 apresenta a comparação entre as áreas das aberturas otimizadas para os efeitos chaminé e vento. Percebe-se que, utilizando o vento no cálculo da vazão, ocorre uma grande diminuição das áreas das aberturas calculadas. Nos casos ilustrados essa redução oscila entre 53,95\% encontrado no método "Frota e Schiffer (2000)" e $65,17 \%$ no método de "Clezar e Nogueira (1999)".

\subsection{APLICAÇÕES DO MÉTODO- SEGUNDA ETAPA}

São apresentados os resultados obtidos a partir da análise das áreas das aberturas em diferentes modelos de pavilhões. Essas análises foram efetuadas com base na literatura técnica e em aplicações da construção civil e buscaram identificar relações entre as dimensões a fim de auxiliar o dimensionamento das edificações.

Nas análises foram considerados pavilhões com larguras de 30, 45 e 60 metros e comprimentos de 60, 90 e 120 metros. Conforme Scigliano e Hollo (2001), o pé-direito do edifício influi diretamente no conforto, pois o efeito chaminé que gera a ventilação natural varia proporcionalmente à variação da altura do prédio. Assim, de acordo com indicações desses autores, as alturas de pé-direito empregadas foram de 6,8 e 10 metros. As dimensões descritas foram combinadas gerando vinte e sete modelos de pavilhões, designados conforme Tabela 3.

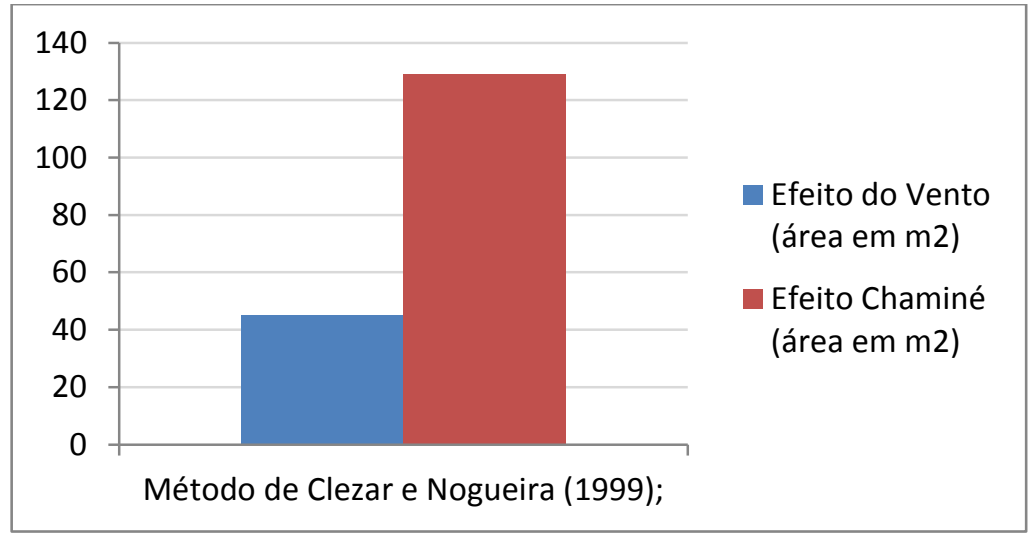

FIGURA 8: Comparação das áreas das aberturas com o uso dos efeitos do vento e chaminé. 


\begin{tabular}{|c|c|c|c|c|c|}
\hline \multicolumn{6}{|c|}{ TABELA 3: Tabela de configurações de dimensionamento dos pavilhões. } \\
\hline $\begin{array}{c}\text { Identificação } \\
\text { do modelo }\end{array}$ & $\begin{array}{c}\text { Dimensões } \\
(\mathbf{m})\end{array}$ & $\begin{array}{c}\text { Identificação } \\
\text { do modelo }\end{array}$ & $\begin{array}{c}\text { Dimensões } \\
(\mathbf{m})\end{array}$ & $\begin{array}{c}\text { Identificação } \\
\text { do modelo }\end{array}$ & $\begin{array}{c}\text { Dimensões } \\
(\mathbf{m})\end{array}$ \\
\hline P01 & $60 \times 30 \times 6$ & P10 & $60 \times 30 \times 8$ & P19 & $60 \times 30 \times 10$ \\
\hline P02 & $60 \times 45 \times 6$ & P11 & $60 \times 45 \times 8$ & P20 & $60 \times 45 \times 10$ \\
\hline P03 & $60 \times 60 \times 6$ & P12 & $60 \times 60 \times 8$ & P21 & $60 \times 60 \times 10$ \\
\hline P04 & $90 \times 30 \times 6$ & P13 & $90 \times 30 \times 8$ & P22 & $90 \times 30 \times 10$ \\
\hline P05 & $90 \times 45 \times 6$ & P14 & $90 \times 45 \times 8$ & P23 & $90 \times 45 \times 10$ \\
\hline P06 & $90 \times 60 \times 6$ & P15 & $90 \times 60 \times 8$ & P24 & $90 \times 60 \times 10$ \\
\hline P07 & $120 \times 30 \times 6$ & P16 & $120 \times 30 \times 8$ & P25 & $120 \times 30 \times 10$ \\
\hline P08 & $120 \times 45 \times 6$ & P17 & $120 \times 45 \times 8$ & P26 & $120 \times 45 \times 10$ \\
\hline P09 & $120 \times 60 \times 6$ & P18 & $120 \times 60 \times 8$ & P27 & $120 \times 60 \times 10$ \\
\hline
\end{tabular}

As vinte e sete configurações resultantes foram aplicadas em três modelos de pavilhões diferenciados pela localização e fechamento de aberturas:

TIPO 01 - Aberturas de parede e cobertura: Modelo de pavilhão com duas aberturas, sendo uma abertura inferior localizada na fachada a sotavento para o ângulo de $90^{\circ}$ e outra abertura superior como exaustor de cumeeira. As variáveis do problema analisadas foram: a altura da abertura inferior, a largura da abertura superior (lanternim) e a altura do peitoril (dimensão mínima igual a $1 \mathrm{~m}$ ).
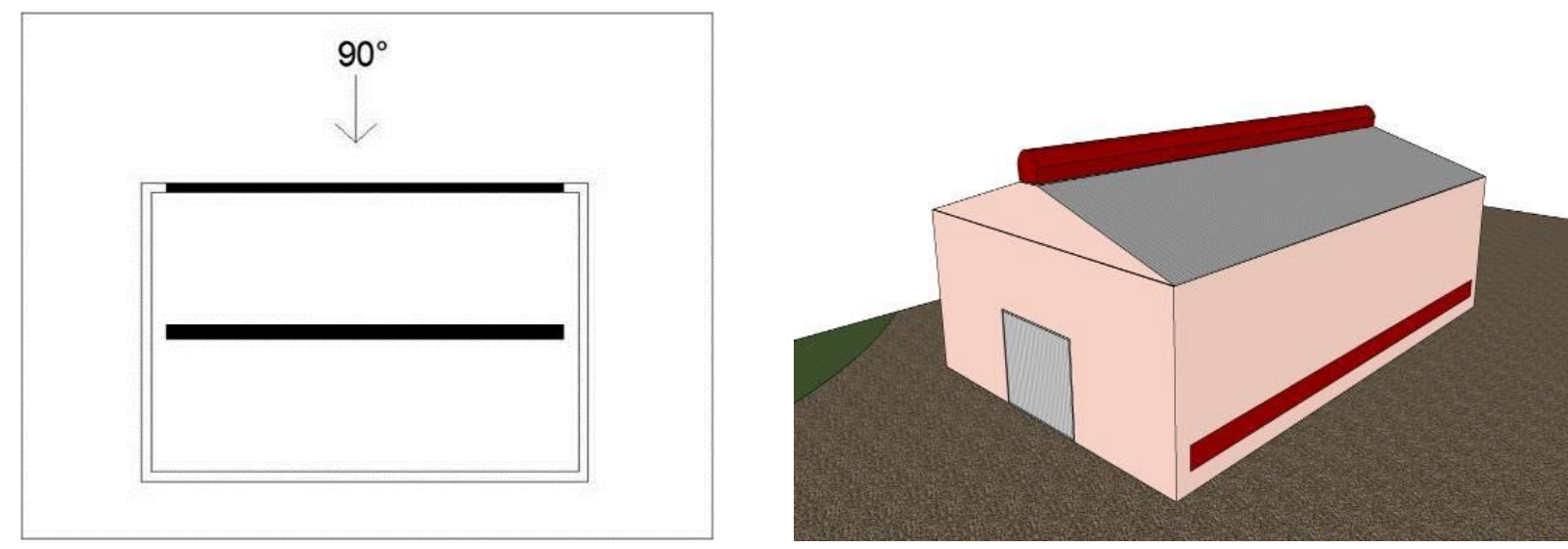

FIGURA 9: Modelo Tipo 1. 
TIPO 02 - Aberturas opostas de paredes: Modelo de pavilhão com duas aberturas localizadas nas fachadas a sotavento e a barlavento para o ângulo de $90^{\circ}$. Analisadas as alturas das aberturas considerando a dimensão mínima do peitoril igual a $1 \mathrm{~m}$.

TIPO 03 - Aberturas opostas de paredes e cobertura: Modelo de pavilhão com três aberturas, sendo duas aberturas localizadas nas fachadas a sotavento e a barlavento para o ângulo de $90^{\circ}$ e uma abertura superior como exaustor de cumeeira. Nesta configuração variam as alturas das aberturas inferiores e a largura da abertura superior (lanternim). Considera-se a altura do peitoril com dimensão mínima igual a $1 \mathrm{~m}$.

Para as análises foram empregadas as planilhas com a metodologia proposta por Clezar e Nogueira (1999) para determinação das vazões.

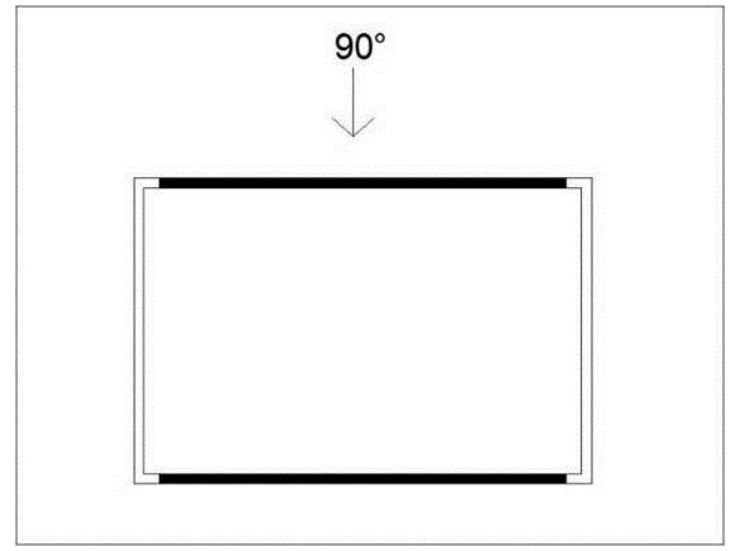

Cada planilha recebeu as dimensões do pavilhão em questão, os dados fixos de temperatura, coeficientes e velocidade do ar além das dimensões e alturas iniciais das aberturas, denominadas como variáveis do problema. A vazão necessária foi determinada com base no volume de cada pavilhão considerando 11 trocas de ar/h. Em função das dimensões relativas, foram determinados os coeficientes de pressão conforme a NBR 6123 (ABNT, 1987).

Para minimizar as áreas das aberturas do pavilhão (função objetivo), estabelece-se as variáveis (altura dos peitoris e aberturas) e restrições (altura mínima do peitoril e vazão necessária com valor próximo da calculada) da formulação. Com a inserção dos dados nas devidas células, o cálculo se dá de forma iterativa permitindo que o suplemento Solver encontre a solução ótima para a função.

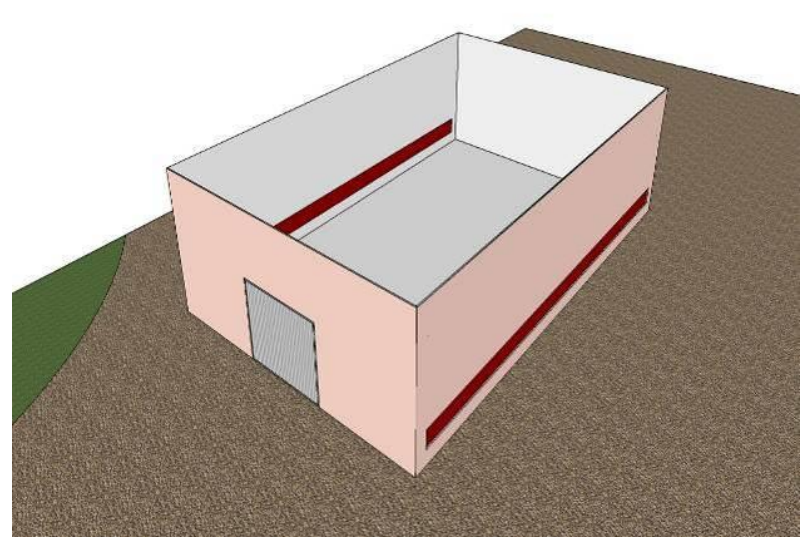

FIGURA 10: Modelo Tipo 2.
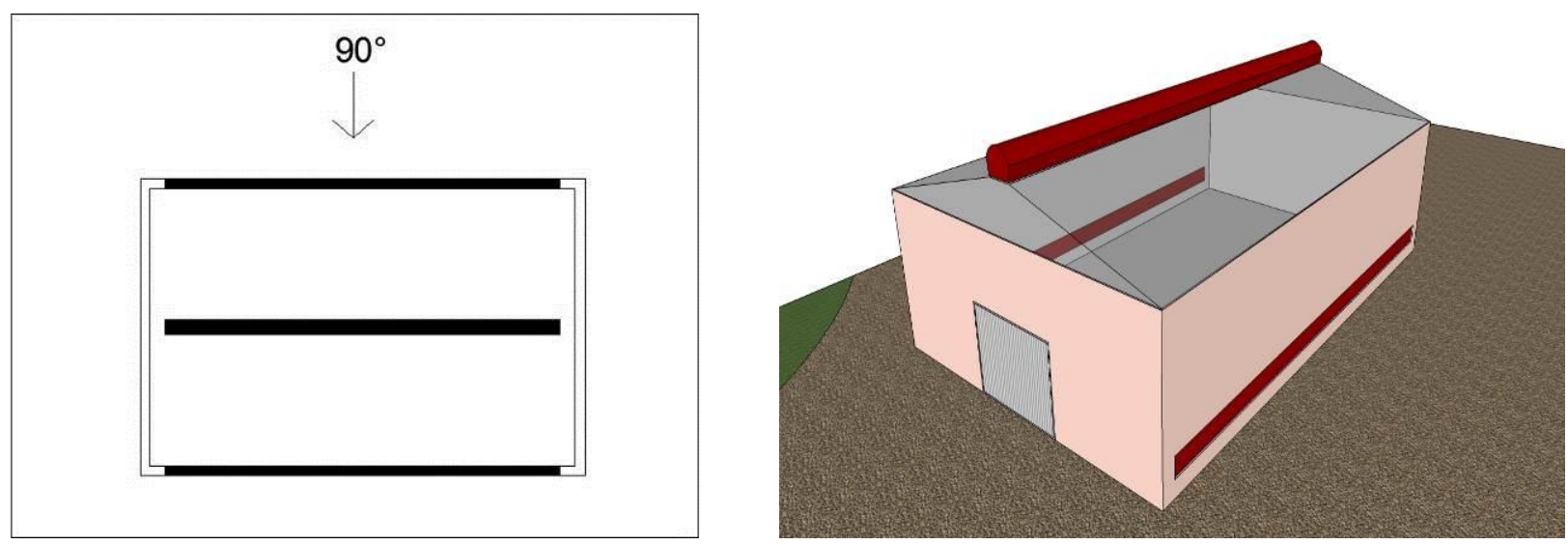

FIGURA 11: Modelo Tipo 3. 


\section{RESULTADOS E DISCUSSÕES}

Para melhor compreensão e visualização dos resultados obtidos na forma como as aberturas e suas posições influenciam o sistema de ventilação, estabeleceram-se três relações entre as áreas encontradas e as dimensões do pavilhão simulado: relação "Área total das aberturas x Área de piso", relação "Área total das aberturas $x$ Volume do pavilhão" e relação "Área de entrada $x$ Área de saída".

Os resultados obtidos evidenciam que a utilização do suplemento Solver do software Excel contribui para a verificação dos objetivos expostos anteriormente sobre a influência que as dimensões e disposições das aberturas exercem sobre a ventilação natural nas edificações.

De forma geral, observou-se que, independente das dimensões dos pavilhões estudados, o efeito do vento gera um aumento significativo na vazão de ar do pavilhão, resultando na diminuição das áreas mínimas das aberturas. Observou-se ainda que, conforme a localização das aberturas nas fachadas, há a possibilidade de se projetar aberturas com dimensões diferentes. 0 uso da abertura superior (lanternim) aumenta a eficiência da ventilação através do efeito chaminé, sendo por isso muito utilizado nesta tipologia de pavilhão industrial. As relações entre as áreas das aberturas encontradas pelo uso das planilhas se diferem quando se considera ou não o uso do vento.

A primeira relação analisada refere-se ao item da área total das aberturas comparada a área do piso do pavilhão. De forma geral a maioria dos percentuais resultantes nesta relação ficou acima do recomendado pelo código de obras de Passo Fundo. Esta diretriz recomenda que o valor desejável para aberturas em pavilhões seja em torno de $4 \%$.

A análise seguinte relata sobre a relação entre a área total das aberturas e o volume do pavilhão, onde se obteve que as variações ocorrem com maior diferença nas dimensões que envolvem o pé-direito de $6 \mathrm{~m}$ e com menor variação no uso de pé-direito de $10 \mathrm{~m}$. Percebe-se que, quando considerado o efeito do vento, os índices referentes a todos os dados de pé-direito possuem valores próximos, sendo as diferenças maiores com a ausência do vento.

Por fim, analisou-se a terceira relação que compreende as áreas de entrada e saída. Utilizando os conceitos encontrados na teoria como embasamento para essa análise, constatou-se que com o uso de vento as áreas otimizadas de entrada e saída ficam com valores próximos entre si. Quando a atuação do vento não é considerada, os resultados obtidos indicaram que a área de abertura de entrada deve ser maior que a área de saída.

\section{CONCLUSÕES}

O presente estudo permitiu verificar a aplicabilidade de técnicas de otimização para minimizar as áreas das aberturas em pavilhões, com o objetivo de melhorar o desempenho térmico da edificação. O modelo teórico de Clezar e Nogueira (1999), baseado na NBR 6123 (ABNT, 1987) - Forças devidas ao vento em edificações, permite calcular a vazão de ar considerando o efeito chaminé, o efeito dos ventos, ou então, ambos os efeitos simultaneamente, por meio de uma formulação única.

As análises efetuadas a partir da metodologia empregada permitiram concluir que:

- Os valores encontrados para relação Área total das aberturas $\mathbf{X}$ Área de piso nos três modelos de pavilhões estão parcialmente de acordo com a proporção estimada pelo código de obras de Passo Fundo, confirmando que este pode ser um bom critério para um pré-dimensionamento das aberturas visando promover uma ventilação eficiente;

- Os valores encontrados para a relação Área de entrada X Área de saída demonstram que a área de abertura de entrada deve ser maior que a área de saída;

- A variação dos índices utilizando os três valores de pé-direito, 6, 8 e $10 \mathrm{~m}$, na relação Área total das aberturas $X$ Volume ocorre de forma parecida, utilizando ou não o vento. Percebe-se 
que quando utilizado o vento, os índices referentes a todos os dados de pédireito possuem valores próximos, diferindo com a ausência do vento.

\section{AGRADECIMENTOS}

Os autores agradecem à Coordenação de Aperfeiçoamento de Pessoal de Nível Superior CAPES pelo apoio financeiro para a realização desta pesquisa.

\section{REFERÊNCIAS BIBLIOGRÁFICAS}

ALLARD, Francis. Natural ventilation in buildings - $A$ design handbook .London, James \& James, 1998.

ASSOCIAÇÃO BRASILEIRA DE NORMAS TÉCNICAS. NBR 15220 Desempenho térmico de edificações. Rio de Janeiro, 2005.

NBR 6123: Forças devidas ao vento em edificações. São Paulo, 1988.

BOUCHLAGHEM, N, Otimização numérica aplicada à concepção térmica dos edifícios. Tese (Doutorado em Engenharia) University of Manchester Institute of Science and Technology, 1990.

CALDAS, L.G; NORFORD, L.K. A design optimization tool based on a genetic algorithm. Automation in Construction, 2002.

CAMPONOGARA, Eduardo. Métodos de otimização: teoria e pratica. Florianópolis: UFSC.2006.

CHEN, Qingyan. Ventilation performance prediction for buildings: A method overview and recent applications. Building and Environment. 1-11, 2008.

CLEZAR, C. A.; NOGUEIRA, C. R. Ventilação industrial. Florianópolis: UFSC, 1999.

FROTA, A. B., SCHIFFER, S. R. Manual de conforto térmico. 2. ed. São Paulo: Studio Nobel, 2001.

FROTA, A. B., SCHIFFER, S. R. Ventilação Natural. São Paulo. 2000.

JEDRZEJUK, H; MARKS, W. Optimization of shape and functional structure of buildingsas well as heat source. Basic theory, Building and Environment, vol. 37, 2002.

ROMERO, M. A. B. Arquitetura bioclimática do espaço público. Brasília, Ed.UnB, 2001.

SCIGLIANO, S., HOLLO, V. IVN - Índice de ventilação natural. 2001. São Paulo: Editora Pini, 2001. 279 p.
SILVANI, M. Subsídios para o projeto da ventilação natural em pavilhões industriais. 2005. Dissertação (Mestrado em Engenharia) - Faculdade de Engenharia e Arquitetura, Universidade de Passo Fundo, Passo Fundo, 2005.

SOLVER, INC. Solver tutorial for optimization users. Disponível em: <http://www.solver.com/tutorial.htm>. Acesso em: 10 jul 2011.

STEPHAN, Louis. et al. Ensuring desired natural ventilation rate by means of optimized openings. 2009. [artigo científico]. Disponível em: <http://www.ibpsa.org/proceedings/BS2009/BS09_228 2_2288.pdf>. Acesso em: 20 mai. 2011.

TOLEDO, E. Ventilação natural das habitações. Maceió: EDUFAL, 1999.

TRINDADE, Sileno Cirne; PEDRINI, Aldomar; DUARTE, Raimundo N. C. Métodos de aplicação da simulação computacional em edifícios naturalmente ventilados no clima quente e úmido. 2010. [Artigo científico]. Disponível em: <http://www. seer.ufrgs.br/ ambienteconstruido/article/view/12110>. Acesso em: 10 jul. 2011. 